\title{
Integração estética entre laminados cerâmicos e coroa total modificada na clínica restauradora
}

\section{Esthetics integration between ceramic veneers and a metal free modified crown in restorative dentistry}

\author{
Mario Cezar Oliveira \\ Alex Correia Vieiral \\ Clístenes Bonfim III
}

\begin{abstract}
RESUMO
Introdução: Restaurar dentes anteriores com estética e previsibilidade é um dos maiores desafios na odontologia contemporânea.

Objetivo: Este relato de caso clínico descreve como alcançar resultados previsíveis com restaurações estéticas em dentes anteriores com laminados cerâmicos associados a uma coroa total metal-free modificada.

Caso clínico: Após realizado o enceramento diagnóstico e mock-up, foram confeccionadas facetas laminadas e uma coroa total modificada com cerâmica à base de dissilicato de lítio. Esse material restaurador apresenta excelente reprodução das propriedades ópticas da estrutura dental, além de alta resistência à fratura. Neste trabalho, todos os detalhes sobre o tratamento foram descritos.

Conclusão: Um resultado estético muito agradável foi conseguido, confirmando que as restaurações adesivas indiretas minimamente invasivas associadas a uma coroa modificada foram excelente opção para o caso apresentado.
\end{abstract}

Palavras-chave: Estética Dental. Laminados. Coroas metal free.

\begin{abstract}
Introduction: Restoring anterior teeth and managing dental esthetics with predictability is one of the most important topics for contemporary dentistry. Objective: This clinical report describes how to achieve predictable outcomes for anterior teeth esthetic restorations with porcelain laminate veneers and a modified dental crown.

Case report: Following diagnostic wax-up and cosmetic mock-up, laminate veneers and full-modified crown were constructed with lithium disilicate porcelain. This restorative material presents excellent reproduction of the optical properties of the dental structure and high-strength. In this paper, the details about the treatment are described.

Conclusion: A very pleasing outcome was achieved, confirming that minimally invasive adhesive restorations associated a modified full crown are an excellent option for these situation.
\end{abstract}

Key-words: Dental esthetics. Venners. Metal free crowns. 


\section{INTRODUÇÃO}

Ao longo dos anos, a estética tem sido primordial no atendimento odontológico. Aliado a isso, vivemos grandes evoluções das técnicas e dos materiais para executar uma reabilitação. Nesse cenário, os laminados cerâmicos e as coroas livres de metal destacam-se como alternativas na escolha do tratamento reabilitador. 1,2,3,4

Para se alcançar um resultado satisfatório com restaurações estéticas, são necessários cuidados em todas as fases do tratamento, que vai desde o diagnóstico criterioso, planejamento, seleção do material, preparos dentais, confecção das restaurações até a cimentação definitiva. Assim a obtenção de translucidez, forma e textura semelhante aos dentes naturais, fornecerá um efeito realista para as restaurações. . $^{3,4,5,6}$

Apesar da existência de materiais com propriedades físicas e mecânicas adequadas para uma boa reprodução da estrutura dentária, ainda é pertinente avaliar, no momento de escolha do material, as condições para a realização de um preparo para a coroa total ou para uma faceta laminada. A quantidade de desgaste dental dependerá de fatores como oclusão e cor do remanescente. Assim, a tomada de decisão de quanto desgastar deve basear-se no quanto será necessário reduzir para atingir o resultado restaurador com função e estética. 1,7,8

Quanto ao processo de fabricação, as restaurações indiretas podem ser confeccionadas de forma convencional ou pela técnica de fresagem a partir de sistemas CAD/ CAM (computer-aided design e computer-aided manufacturing). $\mathrm{O}$ último apresenta vantagens como maior precisão, rapidez e a possibilidade da confecção de coroas e restaurações indiretas de pequena espessura. 8,9,10,11,12

As ligas metálicas são utilizadas nas estruturas para coroas metalo-cerâmicas, porém um dos efeitos indesejáveis dessas ligas é a corrosão que ocorre no meio bucal e o possível escurecimento dos tecidos gengivais adjacentes. ${ }^{13}$ Com a evolução dos materiais cerâmicos, o uso da cerâmica de dissilicato de lítio nas restaurações estéticas tem se difundido devido à alta resistência e excelente translucidez. ${ }^{11}$

Concomitante aos procedimentos estéticos, é necessário manter cuidados com a saúde periodontal, devendo optar-se por um tratamento prévio incluindo raspagem e alisamento radicular. Nesse sentido, para a manutenção da saúde periodontal e menor retenção de placa, as restaurações indiretas, como as facetas de cerâmica, precisam manter um perfil de emergência adequado com boa adaptação às margens gengivais. ${ }^{5,1}$

Via de regra, os cimentos resinosos têm sido utilizados com relativo sucesso clínico para a cimentação de facetas e coroas em cerâmica pura. Esses cimentos, além de fornecerem retenção adequada, deverão uniformizar a cor entre restaurações de espessuras variadas, com diferentes cores de substratos. ${ }^{14}$

Tendo em vista a preocupação com procedimentos de reabilitação que resultem numa boa qualidade estética e que assegurem a manutenção da saúde periodontal, é de suma relevância discutir a aplicabilidade e efetividade da utilização de laminados cerâmicos e coroas de cerâmica pura como alternativas de tratamento na odontologia estética.

A partir disso, tornam-se apropriadas pesquisas que contribuam para a ampliação das discussões científicas acerca desse tema, visto que ainda são escassos na literatura relatos a respeito da utilização concomitante de laminados cerâmicos associados a coroas totais. Dessa forma, este trabalho tem como objetivo apresentar um relato de caso sobre reabilitação estética onde utilizou-se laminados cerâmicos associado a uma coroa modificada em cerâmica pura. 


\section{ReLATO de CAso}

Paciente do gênero feminino de 66 anos de idade procurou atendimento no ambulatório de Prótese da Universidade Estadual de Feira de Santana (UEFS) com queixa estética sobre a cor do dente e gengiva referente ao dente 11 , bem como dos formatos e alinhamento dos elementos 12, 21 e 22 (Figura 1).

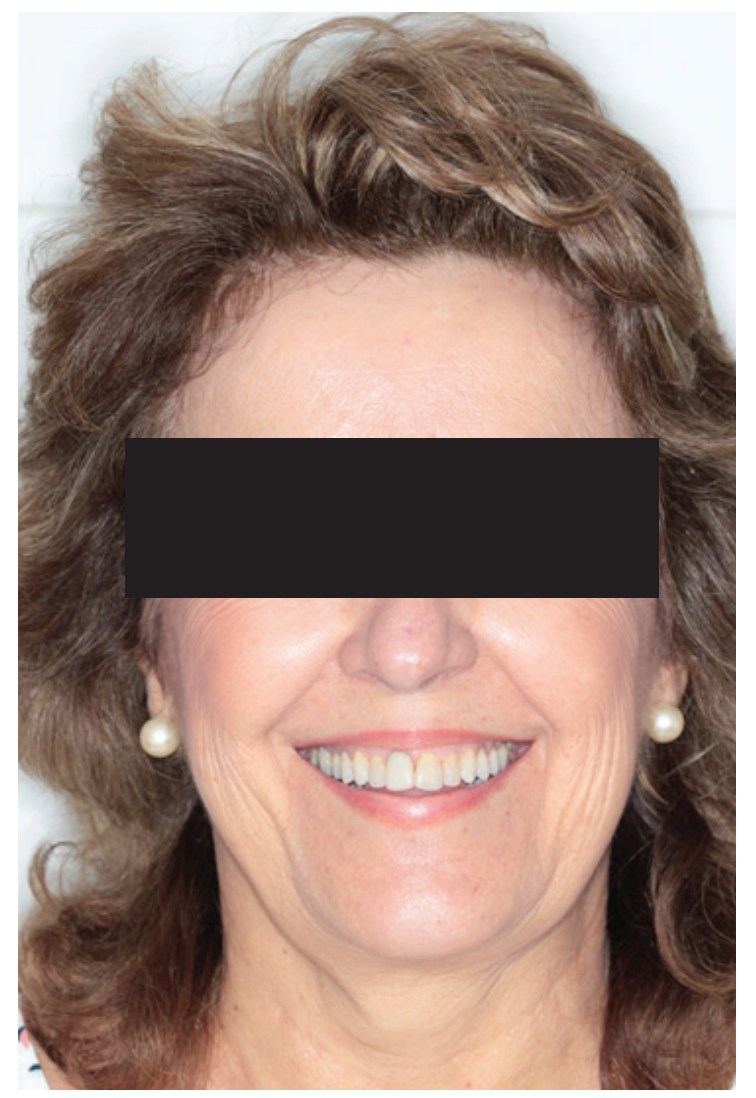

Figura 1: Fotografia inicial da face com sorriso.

Ao serem realizados exames clínico e radiográfico, foram observados os seguintes aspectos: discrepância de cor e anatomia da coroa do incisivo central superior direito, associada à margem gengival escurecida devido à desadaptação marginal, bem como restaurações insatisfatórias nos dentes 12 (face vestibular e mesial), 21 (face vestibular e distal) e 22 (face vestibular, mesial e distal) (Figura 2).

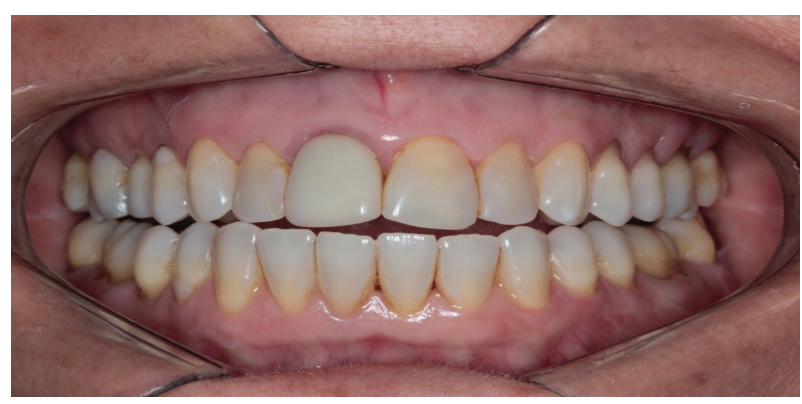

Figura 2: Imagem intrabucal inicial, evidenciando as discrepâncias de cor, formato e alinhamento dos dentes $12,11,21$ e 22.

Após o exame clínico, foram realizados procedimentos de moldagem com hidrocoloide irreversível (Hydrogum, Zhermack, Rovigo, Italy) e confeccionados modelos de estudo para planejamento e enceramento diagnóstico (Figura 3).

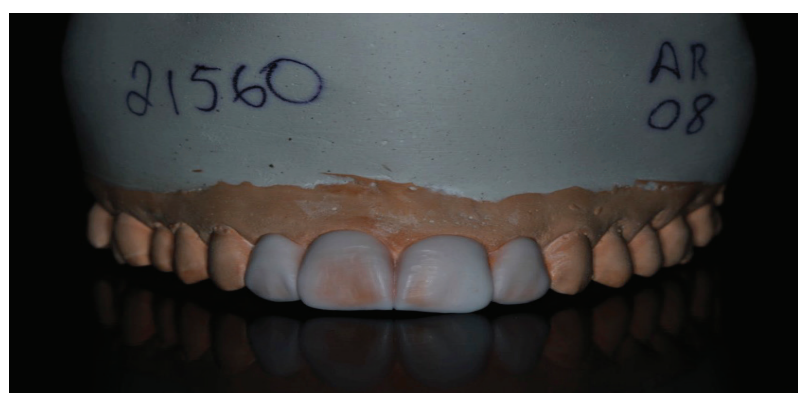

Figura 3: Enceramento de diagnóstico.

Devido às características do caso e ancorado no enceramento diagnóstico, o planejamento estabelecido foi: laminados cerâmicos nas unidades 12, 21 e 22 e coroa em cerâmica pura no dente 11 (IPS E.max, IvoclarVivadent, Schaan, Liechtenstein). A paciente aprovou o plano de tratamento e assinou o termo de consentimento livre e esclarecido.

Antes do tratamento protético, realizouse uma avaliação periodontal seguida de raspagem e profilaxia para a remoção do acúmulo de cálculo e biofilme. A coroa foi então removida e preparos para laminados cerâmicos foram feitos com mínimo desgaste dentário e terminações em esmalte nos elementos 12, 21 
e 22, seguido de preparo para coroa total em cerâmica pura no dente 11. Todos os desgastes foram efetuados com pontas diamantadas (KOMET, Rock Hill, SC, UK).

A partir do enceramento diagnóstico no modelo de estudo, foi confeccionado um molde com silicone de adição (Express, 3M / ESPE, St.
Paul, Minnesota, EUA) e em seguida realizado o mock-up utilizando resina bis-acrílica, cor A2 (Protemp4, 3M ESPE, St. Paul, Minnesota, EUA). Os excessos foram removidos, seguido de ajustes oclusal e estético. Após a realização do mock up (Figura 4), foi confirmado o plano de tratamento proposto.
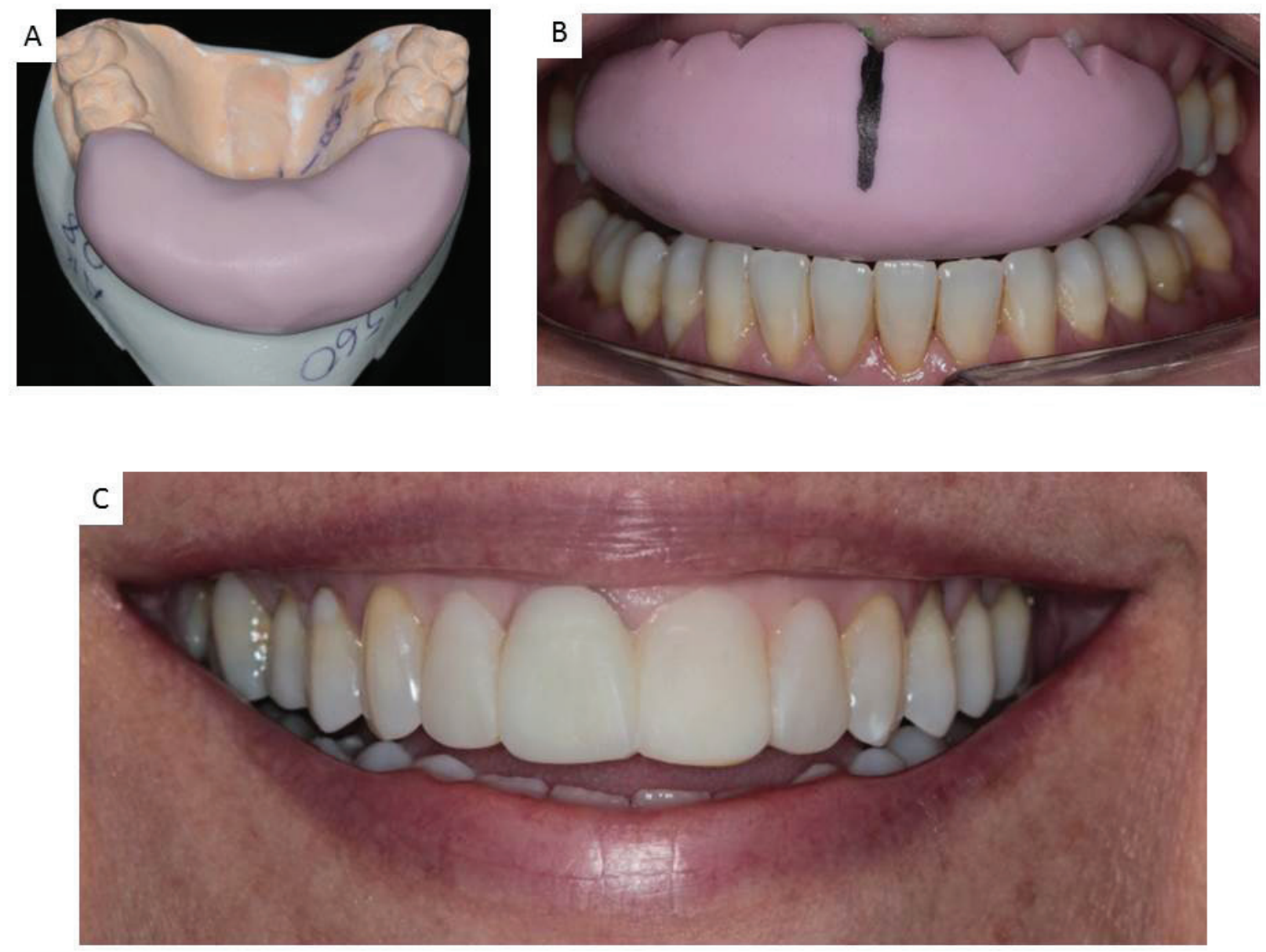

Figura 4: A - Confecção do molde de silicone a partir do modelo encerado. B/C- Mock up com resina bis-acrílica.

Em seguida, foi executadoo procedimento de moldagem pela técnica de impressão simultânea com dois fios de afastamento gengival (Ultrapack, Ultradent, USA) utilizandose silicone por adição (Express, 3M / ESPE, St. Paul, Minnesota, EUA), com posterior seleção de cor e confecção dos provisórios. Fotografias foram enviadas ao laboratório, com os modelos de trabalho e enceramento diagnóstico para melhor comunicação com o técnico em prótese dental, fornecendo ao mesmo as características e detalhes do planejamento proposto.

Devido às características do caso apresentado e para minimizar a diferença de cor entre os substratos dos dentes preparados e a coroa do dente 11, que seriam influenciados pelo agente de cimentação, foram 
confeccionados três laminados cerâmicos para os elementos 12, 21 e 22 e uma infraestrutura em cerâmica pura com faceta estética para ser cimentada sobre a mesma (coroa total modificada) (Figura 5). Em todos os dentes foi utilizada cerâmica à base de dissilicato de lítio (IPS E.max, IvoclarVivadent, Schaan, Lichstetein).
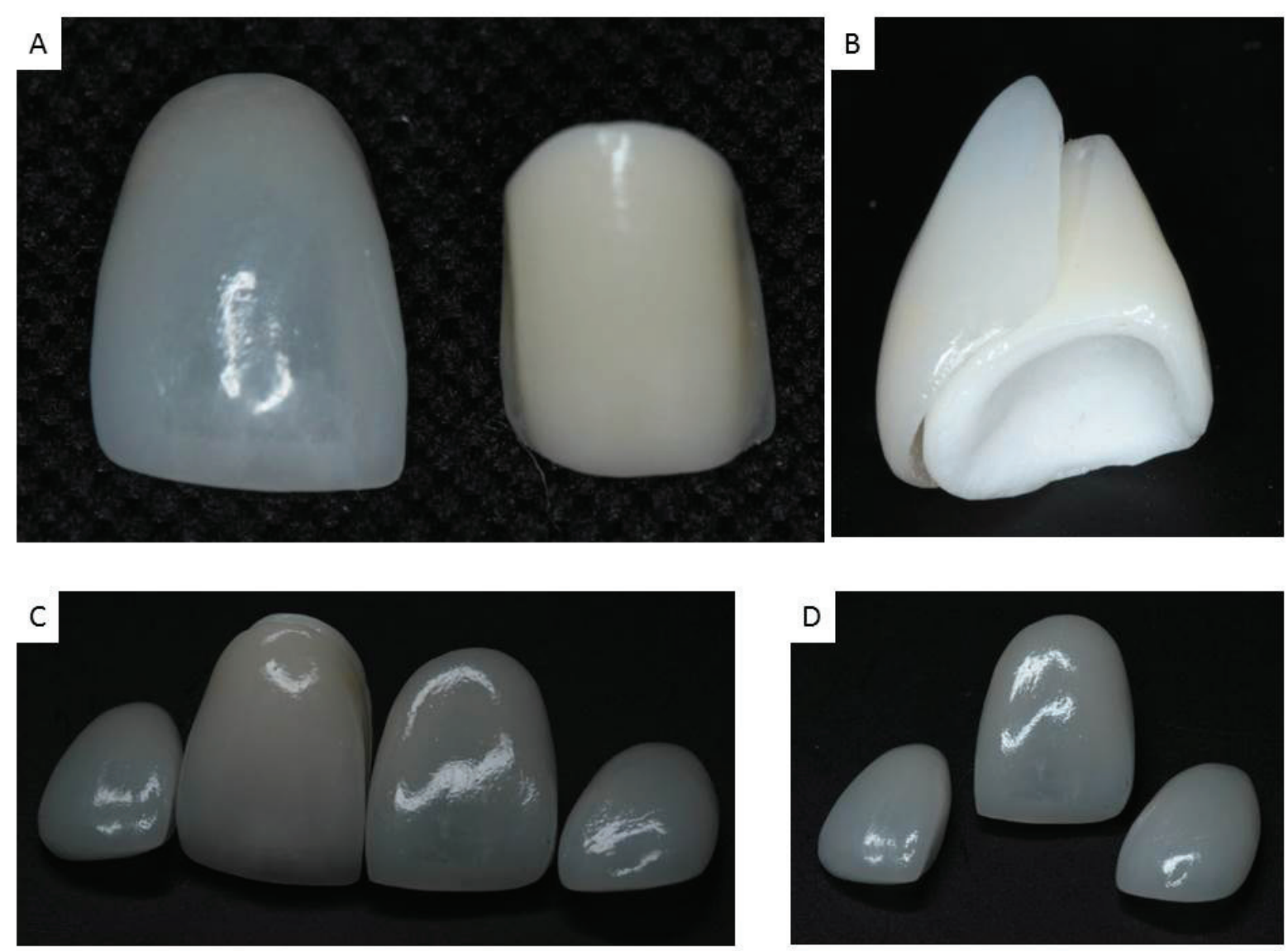

Figura 5: A/B-Vista frontal e lateral da coroa total modificada com sua respectiva faceta estética. C/D- Laminados cerâmicos.

Para a seleção da cor do cimento, realizaram-se testes com amostras de prova "Try-in" (Allcem Veneer Try-in, FGM, Joinville, SC, Brasil), que foram dispensadas na face interna dos laminados cerâmicos e posicionadas sobre os dentes preparados e estrutura da coroa. Após a simulação e escolha da cor do cimento, foi selecionado o cimento da cor A2 para os laminados sobre dentes e cor A1 para o laminado da infraestrutura da coroa.

A cimentação foi então feita com cimento resinoso fotoativado (AllcemVeneer, FGM, Joinville, SC, Brasil). O protocolo de tratamento interno dos laminados cerâmicos e infraestrutura da coroa total foi: 1. Condicionamento com ácido fluorídrico $10 \%$ por 20 segundos; 2 . lavagem com jato de arlágua por 60 segundos e secagem; 3. aplicação de ácido fosfórico a $37 \%$ por 30 segundos; 4. aplicação do agente de união Silano (Prosil, FGM, Joinville, SC, Brasil) por um minuto e secagem com ar; 5. aplicação do sistema adesivo (Adper Single Bond 2, 3M / ESPE, Seefeld, Alemanha) e remoção de excessos com jato de ar; 6 . Fotopolimerização do sistema adesivo. Após realizado o protocolo 
para as cerâmicas, foram executadas as seguintes etapas nos remanescentes dentais: 1. Limpeza dos preparos com pedra-pomes e água; 2. Isolamento com abridor de boca, sugador e fios afastadores 000 (Ultrapack, Ultradent, USA); 3. Tratamento dos substratos dentais com ácido fosfórico por 15 segundos; 4. Lavagem com água/ar e secagem; 5. Aplicação do sistema adesivo (AdperSingle Bond 2, 3M / ESPE, Seefeld, Alemanha) e remoção de excessos com jato de ar.

Em seguida, o cimento resinoso foi dispensado na face interna da infraestrutura da unidade 11 que foi devidamente cimentada sobre o seu remanescente. Após remoção dos excessos de cimento da referida unidade, os laminados foram então cimentados seguindo a escolha prévia de seleção de cor do cimento. Foi usado um microbrush e fio dental nas regiões interproximais dos dentes para remoção dos excessos de cimento, seguido de fotoativação com unidade LED por 60 segundos em cada dente, 30 na face vestibular e 30 na palatina. Os excessos de cimento e fio de retração foram removidos e realizados os procedimentos de ajuste oclusal e polimento com pontas abrasivas para cerâmica.

A obtenção do resultado estético e funcional e a melhora da aparência gengival e harmonia do sorriso são observadas nas Figuras 6 e 7.
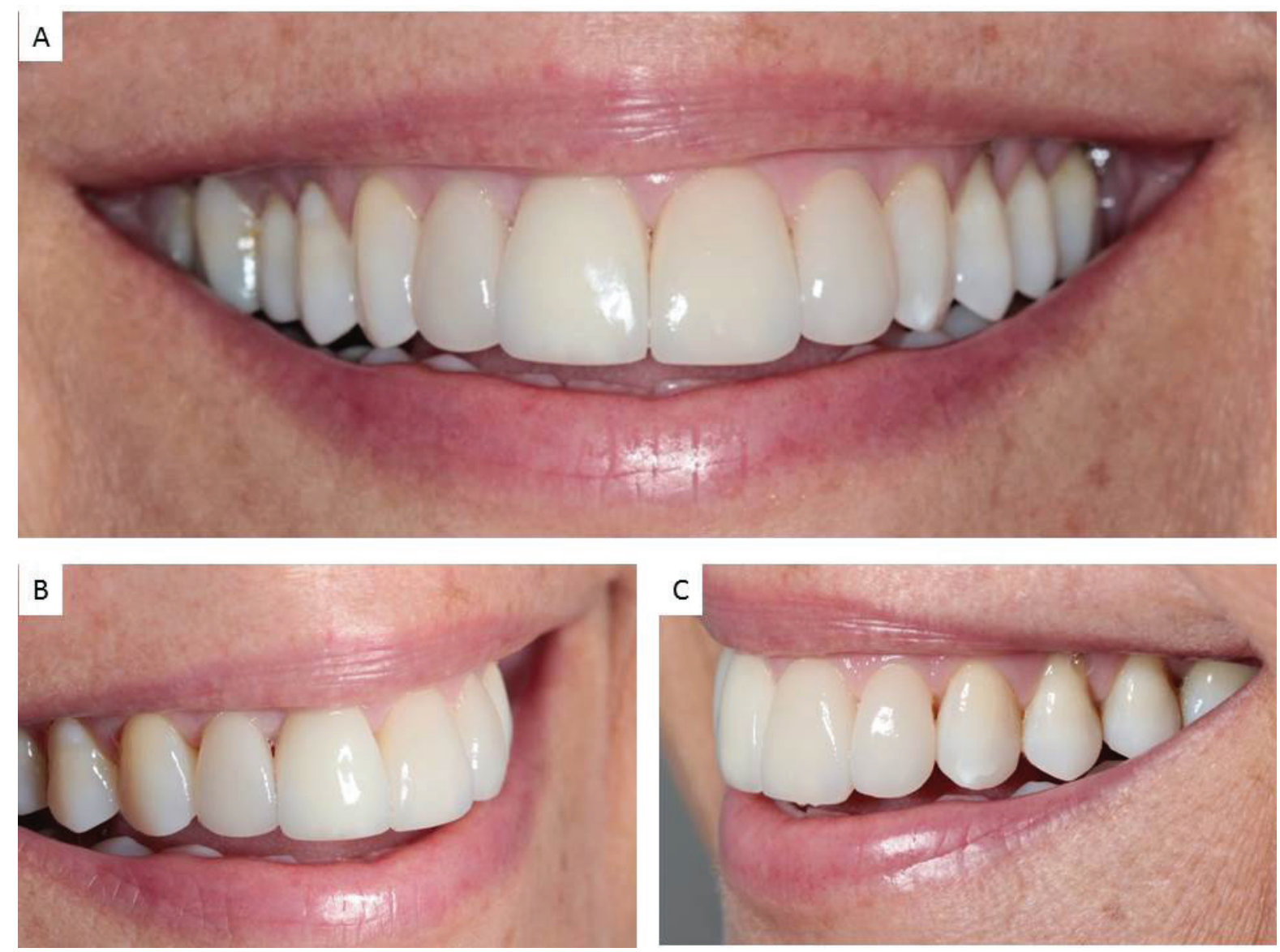

Figura 6: Caso finalizado. 


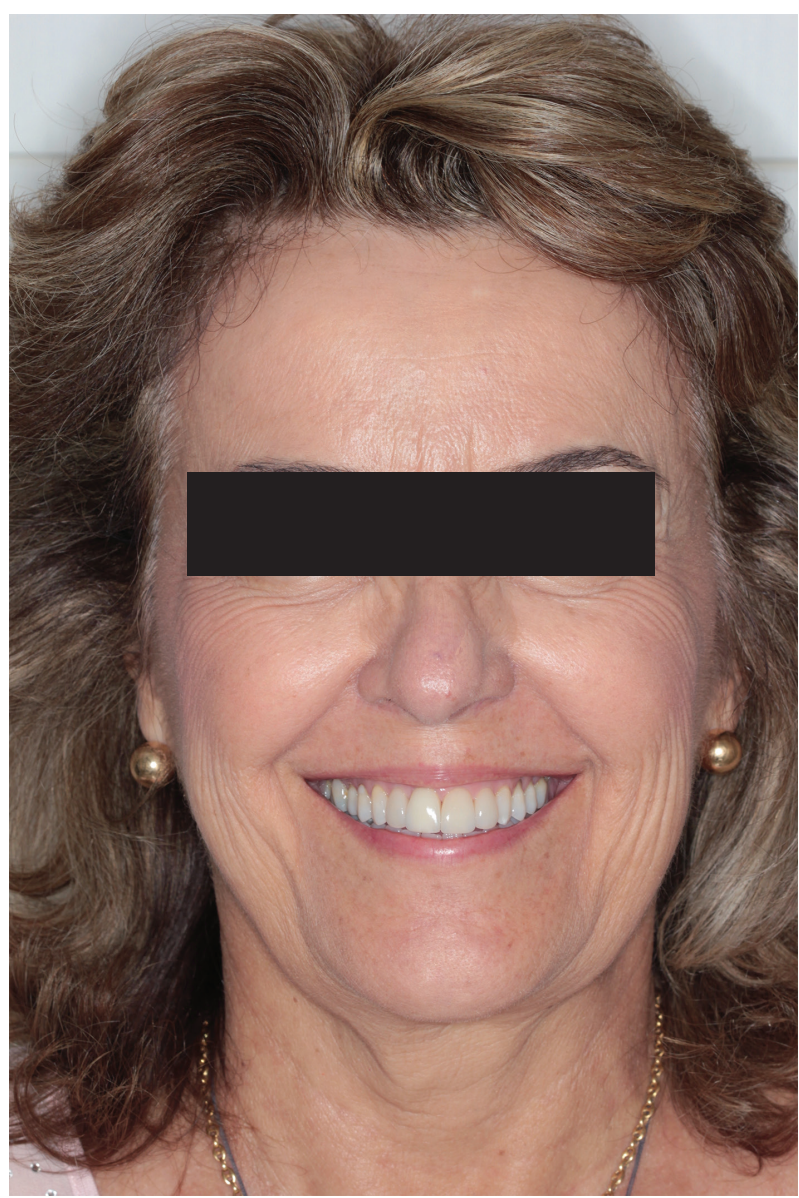

Figura 7: Caso finalizado.

\section{Discussão}

Nesse caso, foi relatado o uso de laminados cerâmicos e coroa de cerâmica pura modificada na reabilitação estética dos incisivos superiores. A opção pelas facetas de cerâmica nos elementos 12, 21 e 22 justificou-se pelo fato de que as facetas de cerâmica podem ser indicadas para o melhoramento estético do formato dental, uma vez que os dentes não apresentem descoloração grave. ${ }^{2}$ Para a resolução estética do dente 11 o tratamento escolhido foi a substituição da coroa por uma coroa de cerâmica pura modificada. A coroa existente apresentava discrepância de cor em relação aos dentes adjacentes e escurecimento da margem gengival.
As coroas de cerâmica pura são indicadas em casos onde já existe grande perda da estrutura dental e a estética é essencial, uma vez que coroas metalo-cerâmicas podem apresentar, ao longo do tempo, escurecimento na margem gengival devido à presença de metal em sua estrutura. ${ }^{1,13}$

Com o objetivo de ter maior previsibilidade do caso, foi realizado enceramento diagnóstico seguido de mockup. A partir desses procedimentos, foi possível redefinir a morfologia dental, alterando forma, comprimento, e posição dos dentes no arco. ${ }^{15}$

A cerâmica à base de dissilicato de lítio foi selecionada para o caso devido às suas propriedades ópticas serem semelhantes às do esmalte dental, além de apresentar boas características de ligação à estrutura dental, desde que seja feito o condicionamento das superfícies internas com ácido fluorídrico associadas a um agente de ligação (silano). Assim, os laminados e coroas de dissilicato de lítio, quando têm suas superfícies internas condicionadas com ácido fluorídrico e recebem aplicação do silano, apresentam uma forte ligação com os cimentos resinosos. ${ }^{11,14}$

Existem evidências de que os cimentos resinosos influenciam na cor final das restaurações com laminados cerâmicos, devido à translucidez e pequenas espessuras dessas peças. Dessa forma, foi utilizada a pasta de cimento resinoso Try-in para selecionar a cor antes da cimentação definitiva, e ter maior previsibilidade do resultado. ${ }^{16}$

Outro fator importante a ser observado em restaurações estéticas indiretas é a cor do substrato. A fina espessura das peças cerâmicas determina que o substrato seja homogêneo, ou o resultado final pode ser comprometido. No caso clínico apresentado utilizou-se, na unidade 11, o artifício de criar substratos uniformes do ponto de vista óptico ao invés de uma coroa cerâmica convencional. Assim, o núcleo metálico fundido na referida 
unidade foi mascarado com uma infraestrutura na qual uma faceta estética seria cimentada numa segunda etapa. Esse recurso possibilitou equalizar a cor do fundo, facilitar os procedimentos laboratoriais e otimizar o resultado estético no momento da cimentação adesiva com $\mathrm{o}$ auxílio do cimento para uniformizar a cor entre a coroa e os laminados.

\section{Considerações finAIS}

Laminados cerâmicos associados a uma coroa modificada em cerâmica estão indicados em reabilitações estéticas, pois permitiram resultados previsíveis e satisfatórios, com melhora na cor, forma e alinhamento dos dentes.

A utilização de uma coroa modificada apresentou vantagens quando existe a necessidade de harmonização da cor entre laminados e coroas totais, visto que existirá a possiblidade de ajustes de cor por meio da seleção de cimentos de diferentes matizes.

\section{REFERÊNCIAS}

1. Cardoso JA, Almeida PJ, Fischer A., Phaxay SL. Clinical Decisions for Anterior Restorations: The Concept of Restorative. J Esth Restor Dent 2012; 24:367-83.

2. da Cunha LF, Reis R., Santana L., Romanini JC, Carvalho RM, Furuse AY. Ceramic veneers with minimum preparation. Eur J Dent 2013; 7:492-6.

3. Sulaiman TA, Delgado AJ, Donovan TE. Survival rate of lithium disilicate restorations at 4 years: A retrospective study. J Prosthetic Dent 2015; 114:364-6.

4. Al Ben Ali A., Kang K., Finkelman MD, Zandparsa R., Hirayama H. The Effect of Variations in Translucency and Background on Color Differences in CAD/CAM Lithium Disilicate Glass Ceramics. J Prosthod 2014. 23:213-220.

5. Cunha LF, Pedroche LO, Gonzaga CC, Furuse AY. Esthetic, occlusal, and periodontal rehabilitation of anterior teeth with minimum thickness porcelain laminate veneers. J Prosthetic Dent 2014; 112:1315-8.

6. Kursoglu P., Motro PFK, Kazazoglu E. Translucency of ceramic material in different core-veneer combinations. The Journal of Prosthetic Dentistry 2015; 113:48-53.

7. Barizon KTK, Bergeron C., Vargas MA, Quian F., Cobb DS, Gratton DG, Geraldeli S. Ceramic materials for porcelain veneers: Part II. Effect of material, shade, and thickness on translucency. J Prosthetic Dent 2014; 112:864-70.

8. Magne P., Carvalho AO, Bruzi G., Giannini $M$. Fatigue resistance of ultrathin CAD/CAM complete crowns with a simplified cementation process. J Prosthetic Dent 2015; 114:574-9.

9. Bagis B., Turgut S. Optical properties of current ceramics systems for laminate veneers. J Dent 2013; 41:24-30.

10. Seydler B., Schmitter M. Clinical performance of two different CAD/CAM-fabricated ceramic crowns: 2-Year results. J Prosthetic Dent 2015; 112:212-6.

11. Fasbinder DJ, Dennison B., Heys D., Neiva G. A clinical evaluation of chairside lithium disilicate CAD/CAM crowns. The Journal of the Am Dental Assoc. 2010; 141:10-14.

12. Boitelle P., Mawussi B., Tapie L., Fromentin O. A systematic review of CAD/CAM fit restoration evaluations. J Oral Rehabil. 2014; 41:853-74.

13. Tian M., Ma S., Niu L., Chen J. Gingival pigmentation by $\mathrm{Ni}-\mathrm{Cr}$-based metal ceramic crowns: A clinical report. J Prosthetic Dent 2016; 115(1):1-4.

14. Christensen GJ, Use of luting or bonding with lithium disilicate and zirconia crowns. J Amer Dental Assoc. 2014; 145(4):383-6.

15. Simon H., Magne P. Clinically based diagnostic wax-up for optimal esthetics: the diagnostic mock-up. J Cafif Dent Assoc. 2008 May; 36(5):355-62.

16. Algahazali N., Laukner J., Burnside G., Jarad FD, Smith PW, Preston AJ. An investigation into the effect of try-in, pastes, uncured and cured resin cements on the overall color of ceramic veneer restorations: An in vitro study. Journal of dentistry $2010 ; 38: 78-86$. 


\section{Dados dos Autores}

\section{Mario Cezar Oliveira}

Graduado em odontologia pela Universidade Estadual de Feira de Santana. Mestre em Odontologia Clínica pela Escola Bahiana de Medicina e Saúde Pública. Doutor em Odontologia pela Universidade Cruzeiro do Sul. Professor Adjunto da Universidade Estadual de Feira de Santana. Feira de Santana/BA - Brasil. mcezar11@ gmail.com

\section{Alex Correia Vieira}

Graduado em Odontologia pela Universidade Estadual de Feira de Santana. Mestrado em Odontologia Clínica pela Escola Bahiana de
Medicina e Saúde Pública. Doutor em Odontologia pela Universidade Cruzeiro do Sul. Professor Adjunto da Universidade Estadual de Feira de Santana. Feira de Santana/BA - Brasil. leko_ vieira@hotmail.com

\section{Clístenes Bonfim}

Graduando em Odontologia na Universidade Estadual de Feira de Santana e Bolsista do Programa de Educação Tutorial - PET-Odontologia UEFS. Feira de Santana/BA - Brasil. cnbomfim@ hotmail.com

Submetido em: 30-06-2016

Aceito em: 28-03-2017 